\title{
Polysèmes
}

Revue d'études intertextuelles et intermédiales

$11 \mid 2011$

Cadres

\section{"Une machine à coudre et à découdre par le fil de l'image » : l'écriture du cadre chez Kay Boyle}

\section{Anne Reynes}

\section{(2) OpenEdition}

\section{Journals}

Édition électronique

URL : http://journals.openedition.org/polysemes/649

DOI : $10.4000 /$ polysemes.649

ISSN : 2496-4212

Éditeur

SAIT

Édition imprimée

Date de publication : 1 janvier 2011

Pagination : $179-200$

ISSN : 0999-4203

\section{Référence électronique}

Anne Reynes, « « Une machine à coudre et à découdre par le fil de l'image » : l'écriture du cadre chez

Kay Boyle», Polysèmes [En ligne], 11 | 2011, mis en ligne le 01 mars 2015, consulté le 02 mai 2019.

URL : http://journals.openedition.org/polysemes/649; DOI : 10.4000/polysemes.649

Ce document a été généré automatiquement le 2 mai 2019.

Polysèmes 


\title{
"Une machine à coudre et à découdre par le fil de l'image " : l'écriture du cadre chez Kay Boyle
}

\author{
Anne Reynes
}

1 Réfléchir aux termes « cadre », « cadrage » et « encadrement » en relation avec l'écriture de Kay Boyle permet de jeter un éclairage intéressant sur une œuvre foisonnante et très contrastée, tout entière fondée sur la recherche d'une forme permettant de frayer un passage vers l'Autre. D'un bout à l'autre de sa carrière littéraire, qui commence dans l'effervescence des avant-gardes parisiennes au milieu des années vingt et se clôt aux États-Unis, son pays natal, dans les années soixante-dix, Boyle fait de l'image la clé de l'accomplissement de cette traversée vers l'altérité. objet de diverses expérimentations empruntant parfois à d'autres modes d'expression artistique, l'image reflète les tensions et les orientations d'une écriture continuée par tous les moyens. De ce fait, elle est investie d'une importance cruciale dans l'œuvre, au point de devenir plus que le moteur de la représentation : l'enjeu d'une prise de position radicale de la part de l'auteur. Or, le mode de fonctionnement de l'image est, chez Boyle, d'emblée lié de manière très concrète à la question du cadre et aux opérations qui s'y rattachent. Dès les premiers poèmes et récits publiés en France, vers 1925, on remarque la présence récurrente de divers objets (miroirs, cadres et châssis de tableaux, cadres de photographies, châssis de fenêtre) dont la fonction « cadrante » est utilisée par l'auteur pour servir d'adjuvant au déclenchement du processus imaginaire. L'objectif est ici d'analyser quelques-uns des procédés auxquels participent ces « objets-cadres » en suivant un itinéraire qui permet d'observer la façon dont l'écriture de Boyle évolue et se transforme au fil de la quinzaine d'années qui sépare son arrivée en France de son retour définitif aux Etats-Unis, notamment sous l'influence des recherches menées autour du photographique et du cinématographique par les artistes et les écrivains associés aux petites revues avant-gardistes auxquelles Boyle collabore également de manière très active. Essayer de comprendre quel rôle joue le cadre dans la poursuite incessante de la forme permet, en effet, de mettre à jour la nature 
d'une pratique particulière et essentielle à l'œuvre de Boyle et, ce faisant, de saisir pourquoi l'image ne peut être chez elle autre chose que l'objet d'un choix radical.

Dans une lettre adressée à William Carlos Williams, quelque temps après son arrivée en France, Boyle s'irrite de ne pouvoir parvenir à donner ce qu'elle appelle une «forme poétique » à son écriture : « Some kind of poetic form has to be found, or I'll go crazy [...] no, no, no form-lousy, loose-no punch-no shape-no agony of line $»^{1}$. Les expérimentations stylistiques et syntaxiques auxquelles se livre l'auteur à cette période expliquent l'urgente intensité de ce besoin de forme. S'inspirant en partie des recherches de Williams, mais également de celles de Gertrude Stein, Boyle s'est engagée dans un travail sur la langue dont le but est de bousculer l'œil dans ses habitudes convenues de lecture séquentielle pour l'amener à percevoir autrement. L'emploi de la répétition, l'ellipse de certaines articulations, le mouvement duel de l'enjambement qui privilégie alternativement la rupture ou la fluidité, le jeu des correspondances synesthésiques comptent parmi les procédés utilisés pour inventer un mode de perception soumis au régime de l'interversion et de l'échange. Le risque, comme Boyle s'en plaint auprès de Williams, c'est que ce travail de brouillage systématique aboutisse à la dissolution totale de la forme et fasse basculer l'écriture dans une parataxe molle et inefficace. La nécessité de retenir la forme à l'intérieur de l'écriture imposant l'intervention d'une figure stable, à même de canaliser le flux des mots et de recentrer l'objet de la perception sous le regard $\mathrm{du}$ lecteur, Boyle choisit alors de se tourner vers le cadre. Un extrait du poème "Summer ", publié dans la revue This Quarter en 1925, permet de démontrer la manière dont elle procède. Ce poème se compose de huit paragraphes en vers libres numérotés avec des chiffres romains et formant autant de vignettes. Dans la seconde de ces vignettes, l'auteur introduit l'image d'une fenêtre qui va venir encadrer l'évocation de ce qui ressemble à un pan de paysage nocturne :

II

I press through the enclosing darkness to the window.

Sky is torn sharp as steel on the yucca horns, clouds pierced

tight as whorls on the yucca horns, plaques of firm flame-

black on black embracing darkness that curve up to sleek

and handsome yucca horns.

Hysteria of the trees is palpable through the closed window

and the wall. The dry tongue of my sheet turns me slowly, tentatively. ${ }^{2}$

En dépit de la présence, au premier vers, de la persona désignée par le pronom «I » à proximité de la fenêtre, le propos du poète ne semble pas être de faire de cette description un "paysage vu d'une fenêtre", mais plutôt d'établir les conditions qui rendent possible la visibilité de ce que les mots décrivent. Si le mot « window ", placé à la fin du premier vers, signale bien l'ouverture d'un espace dans lequel la description peut advenir (v. 2-4), cette ouverture est subordonnée à un mouvement premier qui est celui de la clôture (comme l'indiquent les mots " enclosing », «embracing» et "closed»). À l'équivalence trop simple "fenêtre = paysage ", le poème substitue donc une série d'opérations hiérarchisées que l'on pourrait résumer par la formule : « cadre + fenêtre = paysage ». L'idée que l'acte de tracer un cadre précède et détermine celui de décrire le paysage est encore renforcée aux vers 6 et 7 par le jeu de l'enjambement qui met en exergue la présence du mur sur lequel la fenêtre (qui se révèle d'ailleurs être fermée) se découpe : «Hysteria of the trees is palpable through the window / and the wall ». Ainsi, la fenêtre n'est pas le point de vue depuis lequel il est possible de jouir du spectacle du paysage, mais l'espace même dans lequel ce paysage "noir sur noir» ("plaques of 
flame- / black on black ») va pouvoir se constituer. Le cadre s'inscrit donc dans l'écriture comme un geste premier, qui est à la fois de clôture et d'ouverture. En provoquant le resserrement de l'écriture sur elle-même (ce que soulignent la répétition et la cadence), il force l'image à se rendre visible dans un espace qui n'est ni celui d'un « dedans » auquel la persona semble appartenir ni celui d'un « dehors » situé au-delà du mur et de la fenêtre close. Cet espace " autre », où la vie intérieure de l'image est libre de se déployer, semble plutôt tenir d'une scène rêvée ou fantasmatique, soudain ouverte au regard et à l'imagination du lecteur. En outre, à l'intérieur des limites tracées par le cadre, l'écriture acquiert une qualité tactile qui, à son tour, contamine le mode de perception du sujet appelé « I » dans les trois derniers vers (ainsi que le suggèrent les adjectifs : « Hysteria of the trees is palpable... », " The dry tongue of my sheet... »). Or, il est peut-être possible de voir dans cette prédominance du "sentir ", l'évocation d'un mode d'appréhension du monde qui redouble l'opération accomplie par le geste de l'encadrement. En effet, si l'on s'accorde avec Georges Didi-Huberman qui, à la suite d'Erwin Strauss, considère le sentir comme un mode de connaissance imparfaite en ce qu'il est « un mouvement qui nous porte sans cesse entre contacts et distances $»^{3}$, on est frappé par la proximité entre ce mouvement du sentir et celui de l'encadrement, qui tout à la fois étreint l'image et la maintient à distance. De cette fonction oxymorique du cadre, qui permet le déploiement de l'image dans l'écriture et inaugure un nouveau mode de perception pour le sujet regardeur (ici, la persona comme le lecteur), invité à "sentir» l'image dans son immanence et son opacité, Boyle va faire l'instrument privilégié d'une représentation du sentiment de perte engendré par l'absence.

Après la disparition, en octobre 1926, de son compagnon, le poète américain Ernest Walsh (qui était également l'éditeur de la revue This Quarter), Boyle soumet son écriture à diverses formes d'expérimentation dans le but de trouver un moyen de dire l'absence. Lorsqu'en 1927, Eugene Jolas l'invite à rejoindre le comité de rédaction de la revue Transition, elle se retrouve au cœur d'un projet désormais connu sous le nom du Manifeste de 1929 qui en a énoncé les préceptes : "The Revolution of the Word ». L'objectif de ce mouvement est de parvenir à une transformation radicale de la représentation et du regard en mettant en avant les qualités visuelles et dynamiques du mot. Le cadre et les effets qu'il rend possible jouent un rôle de premier plan dans cette volonté de créer un nouveau langage ouvert aux possibilités proposées par les procédés photographiques et cinématographiques. Car, s'il condamne sans appel le "réalisme photographique » en littérature, Jolas est, en revanche, un fervent admirateur des films d'Eisenstein et de Chaplin, dont il publie plusieurs articles dans Transition, de même que de Man Ray, dont les photographies et les photogrammes extraits des films expérimentaux Emak Bakia (1926) et L'Étoile de mer (1928) paraissent régulièrement dans les pages de la revue. Jolas appelle écrivains et poètes à s'inspirer de ces expérimentations pour créer des formes totalement nouvelles: "The writer has new forms at his disposal, a fusion of forms in which all the senses come into their own [...] the problem of the new form will be the word $»^{5}$. Les réponses proposées par les écrivains associés au projet éditorial de Jolas prouvent l'intérêt qu'ils portent aux expérimentations formelles favorisant l'interpénétration des genres. Ainsi, certains, comme Elliot Paul dans The Ninety and Nine (1928), imaginent des scénarios racontés uniquement avec des photographies. D’autres, comme Henri Poulaille dans Le Train fou (1928), se lancent dans le « roman-film», récit déployant la même rapidité, les mêmes raccourcis et les mêmes montages alternés qu'un film. Jolas, quant à lui, expérimente dans ses poèmes une technique qu'il appelle 
"montage in words", qui rapproche son écriture d'une forme d'art cinématique en utilisant les mots comme des photogrammes, autrement dit en s'inspirant de la division du ruban filmique en cadres ${ }^{6}$. Kay Boyle n'est pas en reste, qui choisit elle aussi de donner à son récit une forme proche de celle du film surréaliste en procédant à «l'hallucination du mot » (« the hallucination of the word »). Avant d'observer grâce à un exemple en quoi consiste ce type d'expérimentation, il est toutefois nécessaire de s'attarder quelques instants sur la relation particulière entre écriture et cadre à laquelle il ressortit.

À partir de 1926, il est très rare d'observer dans l'écriture de Boyle une interaction cadre / image semblable à celle qui a pu être analysée dans "Summer", où le cadre s'inscrit dans l'écriture comme condition première du déploiement de l'image dans la présence. Désormais, le cadre va donner lieu à une autre forme de déploiement, en dedans, vers le vide de l'absence. Il en va ainsi dans Year Before Last (1932), où le recours au cadre souligne l'insurmontable inconnaissance qui régit les rapports entre les êtres. L'intrigue de la première partie du roman est bâtie sur l'absence d'un personnage appelé Eve dont le personnage principal ne parvient pas à se faire une idée claire. Dès les premières pages, l'objet-cadre intervient pour souligner la manière dont l'image se refuse à une lisibilité immédiate. En fait, il s'agit plutôt de deux objets-cadres, le châssis d'un tableau et celui d'une fenêtre, entre lesquels le regard du personnage va et vient :

On the warm January morning which followed, Hannah awoke and the first thing she saw in the room around Martin's bed were the pictures Eve had been painting on the Riviera all winter. [...] the paint rolled off the brush as magnificently as turf heaping the canvas on these few squares that hung around Martin's bed.

Hannah turned her head to the sweet curve of Martin sleeping beside her. Behind him in the window, the sky was clear as a well, and the milk of the clouds flowed steadily out of the strong teats of the southern wind.

Dans les deux cas, le cadre, loin d'accroître la visibilité et la lisibilité des choses, travaille à les rendre indéchiffrables: malgré la surenchère de matière et de couleur, les toiles peintes par Eve n'offrent à voir qu'une découpe sur l'absence. Quant à ce ciel barré d'une blanche coulée de nuages qu'Hannah aperçoit dans la fenêtre, il n'est pas une surface unie et stable, mais mouvante, changeante, soumise au hasard de la météorologie. La course du nuage ne fait que souligner l'illusion d'un hors-cadre qui demeure invisible et renforce le soupçon que cette surface n'est en fait qu'un écran sur lequel l'image vient projeter le mouvement sans fin de son absentement. Le cadre sert ici à produire de «l'en-moins » de présence et de visibilité et, à ce titre, il pourrait être rapproché de ces objets « sans » dont parle Gérard Wajcman dans l'objet du siècle, à propos du ready-made duchampien dont la fonction première est, selon Wajcman, d'exhiber l'absence. Avec beaucoup d'humour, ce dernier se propose d'établir un catalogue des œuvres de Duchamp (ou «entomologie objectale négative») en fonction de ce qui leur manque: selon cette nouvelle classification, la célèbre Roue de bicyclette (1914) est renommée Manque de pneu, le Sèchebouteille (1914), Manque de bouteille, et la pelle de In Advance of the Broken Arm (1915), Manque de neige ${ }^{7}$. Chez Boyle, l'objet "sans » qu'est le cadre fait toutefois résonner le manque de manière plus douloureuse, car il signale une perte irréparable. Le lecteur familier de Year Before Last sait, en effet, que dans les vides que vient forer le cadre se lit non seulement l'absence temporaire d'un personnage mais aussi la prémonition d'une disparition définitive, celle de Martin, qui sera bientôt emporté par la tuberculose (de manière non fortuite, le corps de ce personnage occupe, dans le passage ci-dessus, l'espace dans lequel circule le regard du personnage). Cet extrait du roman de 1932 appelle également une autre remarque, car, outre le fait qu'il démontre clairement la 
manière dont le cadre permet à l'image à la fois de fixer l'absence et de la mettre en mouvement, il souligne le lien que le cadre tisse entre l'œil du spectateur et l'image de l'objet (en l'occurrence, l'image de l'objet en son absence). À travers les yeux d'Hannah, le lecteur est amené, dans le mouvement de va-et-vient du vide au vide, à éprouver le cadre comme ce qui, en même temps, le lie à l'image et le sépare de l'image, autrement dit, pour emprunter cette autre formule à Gérard Wajcman, comme " une machine à coudre et à découdre ": "Machine à coudre le sujet et l'objet par le fil de l'image, machine à découdre en interposant entre le sujet et l'objet, encadrée dans son trou, l'image $»^{8}$. Ce lien entre sujet regardeur et objet regardé est d'une importance capitale, car il détermine la possibilité pour le lecteur de suivre l'image dans son mouvement de repli vers la disparition.

7 Une mise en scène éclairante de la relation du cadre au regard se trouve dans un très court texte intitulé « Vacation-Time », originellement publié en 1928 dans Transition. Sous un titre trompeur, ce récit cherche à dire la perte et la souffrance du deuil en ayant recours au procédé de «l'hallucination » qui repose sur la mise en valeur des qualités optiques du mot grâce à une organisation syntaxique particulière. Le flux de la prose de «Vacation-Time » est ainsi celui d'un "stream-of-consciousness » où mots et groupes de mots sont placés bout à bout sans être interrompus par d'autres marques de ponctuation que le point. Le lecteur est précipité d'emblée dans un courant de sensations et de pensées dont le sens demeure confus. L'effet obtenu est proche de l'effet cinématique expérimenté par Jolas lorsqu'il cherche à utiliser les mots comme des photogrammes : à la lecture, en effet, chaque mot ou groupe de mots ressort isolément de la phrase, tout en demeurant cependant soumis au défilement commandé par le mouvement de l'œil :

And am I now gentlemen we ourselves seeking to outwit the interior to ravage the exterior [...] I am for the gay the biddy a great thing it is to roll home in the furnace of anybody's mouth blasting rust like wine all night and no sleep but the brain too going hot as a black bottom. ${ }^{9}$

L'incertitude concernant le découpage sémantique, ainsi que la possibilité de se livrer à plusieurs montages syntaxiques, conduisent peu à peu le lecteur à abandonner le besoin de " faire du sens » à tout prix pour se laisser aller aux inflexions de la voix narrative et au rythme des images surgissant au fil des mots. Toutefois, cette forme d'écriture hallucinée, qui livre l'accès au plus intime des pensées du personnage, n'est que la première phase de la stratégie narrative mise en place dans la nouvelle. La seconde fait intervenir, à la fin du récit, un objet-cadre qui va soudain dramatiser le sentiment de perte en dialectisant la relation cadre/regard. Il s'agit en fait d'un miroir dans lequel le personnage ne parvient plus à reconnaître sa propre image: "All night I was lying against the mirror because it had a human face to it, lying with my arms around the mirror to soothe the sad old face that was crying in the glass ». L'expérience d'aliénation du personnage (qui est en réalité une très jeune femme) se lit dans l'impossibilité d'identifier comme sien son reflet dans la glace (« it had a human face to it ", « the sad old face»). Dissocié de sa fonction habituelle, le miroir joue ici le rôle d'un mécanisme photographique. Comme dans la construction photographique, en effet, le personnage apparait dans cette scène au miroir comme un sujet qui, s'il est vu, ne peut pas se voir luimême. Son impuissance à voir s'explique sans doute également par l'effet de très gros plan induit par le manque de distance entre son œil et son reflet. Le "cadrage», pour ainsi dire, ne permet pas une bonne lecture de l'image. Le cadre-miroir n'a donc pas ici pour fonction de renvoyer une image, mais de créer un écart dans lequel l'image peut rompre les amarres avec le familier et se mettre à se « dissembler ». Dans cette mesure, 
on pourrait lui appliquer la formule inventée par Marcel Duchamp pour décrire le Grand Verre, œuvre elle aussi basée sur l'idée du dispositif photographique : "C'est miroirique, mais ça n'est pas spéculaire ». Grâce au détour par le photographique, le cadre permet donc à l'œil du lecteur de suivre l'image dans le parcours qui la conduit à s'enfoncer en son dedans, dans l'intimité de ses transformations. Ce pouvoir de métamorphose et de dissemblance dévolu au cadre témoigne du très grand intérêt que Boyle portait aux expérimentations des photographes surréalistes pour provoquer l'effondrement des limites entre réalité et imaginaire, et laisser s'immiscer dans la représentation une (parfois inquiétante) étrangeté. À ce sujet, le rôle joué par le miroir dans la scène d'aliénation de "Vacation-Time» citée ci-dessus n'est pas sans rappeler une photographie de Raoul Ubac intitulée Portrait dans un miroir (même si celle-ci lui est postérieure de dix ans), qui montre le visage d'une jeune femme reflété dans un miroir dont le tain partiellement abymé donne à certains traits un aspect informe et repoussant, alors que d'autres conservent leur netteté. Grâce à cet effet d'asymétrie, le regard peut saisir, au même moment, la beauté et la laideur, la jeunesse et la vieillesse, l'unité et le dédoublement ${ }^{10}$. Jouant de la simultanéité et de l'écart, l'image implique une certaine forme de renoncement. En effet, dès lors que le but poursuivi par les artistes surréalistes est, comme le déclare Man Ray : "to declare cruel contradictions to the adorers of familiar visions $»^{11}$, on comprend que la participation au processus imaginaire oblige le spectateur à accepter le sacrifice de la forme et de la ressemblance. Le même type de déprise est demandé au lecteur de Boyle, qui doit accepter de se plier au fonctionnement du cadre, régi par la perte. Ce qui revient à dire que la perte serait au fond la forme à travers laquelle l'écriture de Boyle parvient le mieux à transiter vers l'autre, dans ce mouvement d'écart opéré par le cadre, qui porte l'image à s'éprouver dans sa propre altérité. Cette idée permet également de mieux cerner la nature spatio-temporelle du cadre.

9 Jusqu'ici, j'ai employé, pour désigner le cadre, les termes de «surface » ou " d'espace », mais il s'agit à présent de définir plus précisément « où » le cadre se tient dans l'écriture de Boyle. Et pour cela, revenir une nouvelle fois sur son mode opératoire, mais par un autre biais. Comme on a pu déjà l'observer, le cadre est associé chez Boyle à un type d'images que l'on peut qualifier de "récalcitrantes » à la représentation : tableaux à la figurabilité indéfinissable - ou dont les toiles sont retournées contre les murs, cadres orphelins de leur portraits photographiques, miroirs trompeurs, l'œuvre multiplie les ruses pour mettre en déroute les images trop facilement identifiables. En outre, s'il cherche à délimiter un espace pour y concentrer le regard, le cadre n'a pas pour objectif d'apporter un surcroît de visibilité, mais, au contraire, de provoquer cet écart qui va conduire l'image à s'altérer, c'est-à-dire à devenir autre en se transformant. Le cadre ressortit donc d'une double logique qui est celle de la contrainte (clôture) et de l'accidentel (moment du passage vers l'altérité). Il est ainsi le signal de l'immanence d'un événement marqué par le glissement de l'image vers la disparition et l'irruption du sentiment d'absence. Dans une nouvelle de 1933 intitulée " I Can't Get Drunk », qui narre l'errance angoissée de deux amis, la nuit, à Paris, de bar en bar, le flot fiévreux de l'écriture est ainsi interrompu lorsque les deux personnages se rendent compte que le miroir faisant face à la table de café à laquelle ils ont pris place ne renvoie plus l'image de leurs visages, mais le reflet d'un glissement, d'un écoulement dans l'informe :

There's something happening somewhere else he said. Into these places and out of them we went. There's something happening on the other side of town said Denka. We stepped into a taxi to track it down. Before the café mirror we sat still and 
surveyed the landslide of our faces down the glass.

Your appearance is such a deceptive one I said. ${ }^{12}$ que Man Ray réalise à la même période en expérimentant le processus de solarisation du négatif, démontre à nouveau le rôle déclencheur du cadre dans le surgissement de l'accident à la surface de la représentation, qui permet ensuite à l'image de se déployer vers la profondeur de l'absence. Ce déploiement peut parfois apporter à l'image la possibilité de se revitaliser, comme c'est le cas dans «I Can't Get Drunk », où l'apparition du miroir marque le moment charnière où l'image, désormais libérée de l'obligation de ressembler, va subir une série de métamorphoses jubilatoires. Dans la suite de la nouvelle, le lecteur découvre le récit décousu et délirant de souvenirs que les deux personnages se remémorent. Même si l'objet de l'angoisse existentielle du personnage appelé Denka demeure non-dit, une revigorante inventivité est insufflée à la parole :

We wouldn't lie still for the moonlight I said it wouldn't let you sleep Nor would it said Denka.

We sat up all night with it, holding its hands and saying kind things to it. We rolled it up into a rug and told it stories in its ears.

Associant rupture et mouvement pour permettre le dégagement de l'écriture dans l'imaginaire, le cadre parait, par conséquent, correspondre à cette définition donnée par Julia Kristeva pour désigner « une articulation toute provisoire, essentiellement mobile, constituée de moments et de stases éphémères " ${ }^{13}$, qu'elle appelle la « khôra ", autrement dit le «lieu ». Extensif de par sa relation au «fond sans fond » vers lequel il permet à l'image de se mettre en mouvement, le cadre est donc également lié au moment de défaillance qui vient faire basculer la représentation. Toutefois, sa nature temporelle est double, puisqu'il participe également d'une mémoire de la perte qu'il permet d'articuler dans l'écriture. Le cadre serait donc finalement ce qui appartient à un temps sans fin et à une surface sans fond, ce «lieu» où l'image peut venir représenter la perte. Faire du cadre le « lieu » de l'image chez Boyle n'explique néanmoins pas toute l'importance qu'il a au regard de l'œuvre. Il est donc nécessaire de pousser la réflexion un peu plus avant en examinant ce qui fait plus précisément son pouvoir en tant que lieu.

Sa double fonction de rupture et d'articulation indique ce qui fait l'essence du cadre dans l'écriture de Boyle, je veux parler de sa nature profondément rythmique. En tant qu'il est, comme on a pu le voir, l'une des modalités de l'écart dans lequel le mouvement imaginaire peut se produire, le cadre contribue à instaurer une oscillation essentielle. C'est, en effet, dans l'écart que l'image peut s'absenter au-dedans d'elle-même, à la rencontre de son propre inconnu, pour y trouver de nouvelles formes. Du point de vue du lecteur, faire l'expérience du cadre, c'est faire l'expérience de cette oscillation de l'image entre présence et absence, de ses départs vers la profondeur. C'est donc également prendre part au jeu de la perte et de la réappropriation, puisque c'est en acceptant l'idée que le dessaisissement est la condition première du déclenchement du mécanisme imaginaire que le lecteur peut, en retour, participer pleinement au spectacle des transformations de l'image. Or, ce jeu, comme l'a expliqué Nicolas Abraham, ressort d'une activité créatrice «rythmisante». Je rappellerai ici simplement que, pour Abraham, le rythme n'appartient pas à l'objet, mais n'existe que dans la conscience que le sujet en a. Avoir conscience du rythme, c'est anticiper un retour, attendre « un nouveau futur ». En tant que pratique créatrice, la «conscience rythmisante » permet donc l'exercice d'un certain pouvoir démiurgique : 
Pour elle, l'avenir ne se définit pas par les catégories du connu ou de l'inconnu. Elle n'observe ni ne prévoit. Ce qui arrive arrive par sa propre vertu à elle. Son avenir est décisoire. Elle se crée en créant le monde. [...] L'accomplissement du retour attendu sera l'éclatante confirmation de son pouvoir d'abord mis en péril. Ainsi, en tant qu'elle est agissante, la conscience rythmisante a toujours le dernier mot. ${ }^{14}$ D'abord, parce que, vecteur d'écart et d'espacement, il est un moteur du mécanisme oscillatoire de l'image. Mais également, parce que chacune de ses apparitions dans le texte, qui signale l'événement de l'ouverture de l'image à l'altérité, produit à la longue une anticipation qui implique directement le lecteur dans le processus d'écriture et accroît le plaisir qu'il tire du texte. Chercher à savoir jusqu'où Boyle a poussé cette "pratique rythmisante» de l'écriture du cadre permet de prendre la mesure de son importance au sein de l'œuvre.

38, au terme d'une décennie d'expérimentations diverses qui lui ont gagné l'estime des éditeurs et des critiques, Boyle publie un recueil de trois récits intitulé Three Short Novels dans lesquels l'image semble faire l'objet d'un doute radical, comme le montre cet extrait de "The Bridegroom's Body» qui décrit ce qu'aperçoit un personnage du haut d'une falaise qui surplombe la mer :

It might have been a detached universe she saw below as if static under glass. There was the far line of the shingles on which the seemingly motionless surf continuously broke, the white thread-like edge of foam as if painted there by hand; and the single gulls that might set out in flight, so farly seen that they were birds no longer but chalk-like splinters falling in retarded motion towards the water bleak gray becalment. ${ }^{15}$

Bien que le texte ne mentionne pas de manière explicite la présence d'un cadre, cette description est clairement présentée comme une mise en tableau du paysage. L'objet de la vision, qui apparaît «détaché » du reste du monde, semble maintenu sous un film de verre ("as if static under glass »). La répétition de la locution "as if », de même que l'adverbe "seemingly ", établissent également une nette séparation entre l'espace dans lequel se tient le personnage-spectateur et l'espace de la représentation. Cette idée de séparation est enfin accentuée par l'utilisation de "farly ", mot qui ne possède pas de réalité lexicale ni grammaticale, et qui contribue donc à introduire un nouvel écart dans le texte. Quant à la représentation proprement dite, elle se réduit à un tracé de fines lignes horizontales ( the far line of the shingles », « the white thread-like edge of foam ») surmontées de petits traits isolés. La dominante chromatique de cette composition est le gris de la mer et du rivage sur lequel se détache le blanc de l'écume et du plumage des mouettes. Autrement dit, un gris-blanc qui tire vers le monochrome. La profondeur de champ, comme le souligne la répétition du mot "far ", paraît infinie et contribue à la transformation de l'image en un espace illimité et quasiment vide. L'absence de bordure se lit comme une entorse à la rythmique du cadre à laquelle Boyle avait jusque-là habitué son lecteur. Il semble, en effet, que l'écriture se mette d'un coup, sans prévenir, à se vider de ses images pour se changer en une sorte de paysage "blanc sur blanc » n'offrant quasiment plus rien à voir. Une fois disparues les limites du cadre, reste une frustration sans fin du regard devant l'épuisement également sans fin de l'image dans un espace vide. Cette volonté d'aller jusqu'au bout du mouvement d'abstraction de l'image dans l'absence doit-il s'interpréter comme une tentation du sublime ? C'est là une lecture possible, mais elle ne prend pas en compte le fait que si l'écriture ne mentionne pas l'existence d'une bordure à ce tableau-paysage, elle suggère en revanche la présence d'un châssis, en 
insistant sur la matérialité de son support. C'est là ce que signale le curieux rapprochement entre les mouettes et les « chalk-like splinters ». Le mot " chalk » évoque bien entendu la couleur blanche, mais il renvoie également à la craie dont on se sert pour tracer des signes sur une surface. Quant au mot «splinters ", il désigne un picot saillant sur une surface en bois. D'où une autre lecture possible de cette description, non plus comme un vide sans fond où l'image n'en finit pas de s'absenter, mais comme une surface d'inscription hérissée d'échardes où l'œil court le risque de s'écorcher. Mouvement vers le sublime tout autant que brutale désublimation, l'image est ici à la fois ce qui est irrémédiablement perdu et ce sur quoi le lecteur vient brutalement cogner de l'œil. Dans les deux cas, la rythmique du cadre s'est enrayée, provoquant l'arrêt de la dynamique qui animait l'image. Au vu de l'importance accordée par Boyle à la mise en place de cette rythmique particulière, on devine que ce choix ne peut relever que d'une impérieuse nécessité, ou de circonstances extraordinaires.

En effet, la publication de Three Short Novels coïncide avec la décision de l'auteur de repartir aux Etats-Unis et d'abandonner la forme littéraire pour la chronique journalistique. Boyle donne à ce choix une raison politique. Au cours des années trente, divers séjours dans plusieurs pays d'Europe, notamment en Autriche, lui ont permis d'assister de près à la montée des fascismes et aux prémisses de la deuxième guerre mondiale. Très tôt, elle se dit persuadée que seule la participation des États-Unis au conflit peut sauver l'Europe du gouffre, et déclare que sa décision d'abandonner la forme poétique qui lui est familière relève du désir de s'adresser au plus grand nombre par le canal le plus pratique. Le choix du politique contre l'image masque toutefois un doute plus fondamental au sujet de cette dernière, qui semble ne plus pouvoir fonctionner selon le mécanisme oscillatoire qui assurait auparavant son libre déplacement de forme en forme. Il n'est pas possible d'analyser ici les raisons de cette « crise de foi » en l'image, qui conduit l'auteur à la laisser disparaître dans le paysage passé à blanc du récit de Three Short Novels. Cependant, un constat s'impose : jamais plus l'image ne retrouvera une force d'inventivité créatrice semblable à celle qui animait les poèmes et les récits des années vingt et trente. Et jamais plus le cadre, redevenu, dans la prose des romans publiés dans les années cinquante, un banal objet du quotidien, n'aura la fonction opératoire qui en faisait le «lieu privilégié » du processus imaginaire deux décennies auparavant. À une exception près toutefois.

Dans une série de poèmes écrits au début des années soixante et regroupés sous le titre «Poems for a Painter $»^{16}$, qui traitent précisément de la difficulté de la création artistique, l'écriture de Boyle, un moment réenchantée, retrouve le rythme qui lui était autrefois propre. Le poème intitulé « Print from a Lucite Block », dont je cite ci-dessous la première et la dernière strophes, permet de s'en rendre compte. Il se construit dans la tentative de rendre l'impression produite par une vision éphémère, aperçue à travers un matériau translucide et très opaque difficile à identifier, nommé " glace » (« ice ») dans la première strophe et "verre» ("glass») dans la dernière. Si elle ne peut capturer cette image, l'écriture parvient néanmoins à évoquer le moment où elle transite entre absence et présence, obscurité et clarté :

Last night, as I crossed

The black ice toward your image

A deer ran.

[...]

In the night's cracked glass 
Your image turned from shadow to moonlight

As the antlered deer leapt past. matériau de la représentation («the night's cracked glass»). En l'absence d'un cadre explicitement convoqué pour venir cerner le moment d'échappée dans lequel l'image se laisse un instant appréhender, il est peut-être possible de voir dans cette petite fissure qui troue la surface de la représentation comme un retour à la condition première de la vision, une sorte de cadre archaïque (le "spiracolo" dont Léonard de Vinci fait la condition de l'exercice de la faculté visuelle $\left.{ }^{17}\right)$. Ce que le cadre rend possible dans ce «Poem for a Painter », et on mesure par là son importance au regard de l'œuvre tout entière, c'est une forme de "voir à travers " comme moyen d'inventer une "passe " essentielle pour s'ouvrir vers l'Autre et provoquer l'apparition du monde. Ce qui fait du cadre chez Boyle, le « avec » d'une écriture avec l'image, ou, plus encore, le «par » d'une écriture par l'image, continuée, par-delà ruptures et doutes, malgré tout.

\section{NOTES}

1. The Selected Letters of William Carlos Williams, John C. Thirwall (ed.), New York: Mac Dowell, Obolensky, 1957, 129.

2. Kay Boyle, "Summer", This Quarter 1 (1925): 40-42.

3. Georges Didi-Huberman, Génie du non-lieu, Paris : Minuit, 2001, 145-146.

4. Proclamation en douze points publiée en juin 1929 dans la revue Transition et signée par seize écrivains parmi lesquels Hart Crane, Harry Crosby, Stuart Gilbert, Eugene Jolas et Robert Sage. Ce manifeste déclare, entre autres, le droit à l'autonomie totale de l'auteur (« The imagination in search of a fabulous world is autonomous and unconfined »), qui doit chercher à métamorphoser la réalité en "l'hallucinant ", à travers la poursuite d'une "poésie pure " dégagée de toute attache référentielle ( Pure poetry is a lyrical absolute that seeks an a priori reality within ourselves alone », « Time is a tyranny to be abolished »).

5. Eugene Jolas, “Towards New Forms?” Transition 19-20 (1930): 104.

6. La «Reading Machine " inventée en 1931 par Robert Carlton (Bob) Brown représentera un aboutissement logique à ce projet d'écriture cinématique de Jolas. Cet appareil portable, composé d'un ruban défilant sous une loupe, avait pour objectif d'isoler les mots de leur contexte sémantique et syntaxique, et de les animer d'un mouvement mécanique permettant au lecteur de faire de la lecture une expérience similaire à celle procurée par le spectacle d'un film.

7. Gérard Wajcman, L'Objet du siècle, Paris : Verdier, 1998, 82-83.

8. Gérard Wajcman, Fenêtre. Chronique du regard et de l'intime, Paris : Verdier, 2004, 187.

9. Kay Boyle, Wedding Day and Other Stories, New York: Jonathan Cape and Harrison Smith, 1930.

10. Cette photographie de Raoul Ubac (visible sur images.google.fr) est citée par Rosalind Krauss dans son analyse des procédés photographiques surréalistes in Le photographique. Pour une théorie des écarts, traduit de l'américain par Marc Bloch et Jean Kempf, Paris : Macula, 1993.

11. Man Ray, "Deceiving Appearances", Photography in the Modern Era, Christopher Phillips (ed.), New York: Aperture, 1989, 12.

12. Kay Boyle, “I Cant' Get Drunk", Life Being the Best and Other Stories, New York: Harrison Smith and Robert Haas, 1933. 
13. Citée par Anne Cauquelin in Le Site et le paysage, Paris : PUF, 2002, 81-82.

14. Nicolas Abraham, «La conscience rythmisante : essai sur la temporalité du rythme » (1952), Rythmes : de la philosophie, de la psychanalyse, de la poésie, Paris : Flammarion, 1985, 104.

15. Kay Boyle, Three Short Novels, Boston: Beacon Press, 1958.

16. Kay Boyle, Collected Poems, New York: Alfred A. Knopf, 1962, 99-104.

17. Voir Hubert Damisch, L'Origine de la perspective, Paris : Flammarion, 1987, 148.

INDEX

oeuvrecitee Life Being the Best and Other Stories, Three Short Novels, Wedding Day and Other

Stories 